\title{
Cofactor dependence and isotype distribution of anticardiolipin antibodies in viral infections
}

\author{
H Guglielmone, S Vitozzi, O Elbarcha, E Fernandez
}

\begin{abstract}
Background-Antibodies to cardiolipin (aCLs) are often detected in patients with autoimmune disorders or infectious diseases.

Objective-To investigate the distribution of aCL isotypes and requirement of protein cofactor in viral infections in order to establish the importance, if any, of these antibodies in these infectious diseases.

Patients and methods-The isotype distribution of aCLs in the sera from 160 patients with infection caused by HIV-1 $(n=40)$, hepatitis A virus $(n=40)$, hepatitis B virus $(n=40)$, or hepatitis $C$ virus $(n=40)$ was studied by standardised enzyme linked immunosorbent assay (ELISA) in the presence and absence of protein cofactor (mainly $\beta 2$-glycoprotein I). Serum samples from healthy volunteers and patients with syphilis and antiphospholipid syndrome were also included and served as negative and positive control groups respectively.

Results-The prevalence of one or more aCL isotypes in serum of patients with HIV-1, hepatitis A virus, hepatitis $B$ virus, or hepatitis $C$ virus infection was $47 \%$, $92 \%, 42 \%$, and $17 \%$ respectively (principally IgM and/or IgA). Most of these antibodies were mainly cofactor independent. Conclusions-The presence of aCLs in viral infections is principally cofactor independent, suggesting that cofactor dependence of the aCLs should be assessed to distinguish subjects most likely to suffer from clinical symptoms observed in the presence of these antibodies.

(Ann Rheum Dis 2001;60:500-504)
\end{abstract}

Lupus anticoagulant (LA) and antibodies to cardiolipin (aCLs) are acquired antibodies to phospholipid (aPLs) which may be present in a variety of conditions. Classically, they are found in patients with systemic lupus erythematosus (SLE) or related autoimmune disorders but they may also be detected in patients with syphilis and a variety of acute or chronic infections. ${ }^{12}$ Certain people, however, have no identifiable underlying disorders and may be regarded as having primary antiphospholipid syndrome (APS). ${ }^{3}$ Clinical signs linked to aPLs usually involve thrombosis, recurrent fetal loss, and thrombocytopenia which occur in about $30 \%$ of cases; less common conditions such as livedo reticularis, leg ulcers, chorea, epilepsy, and pulmonary hypertension are also involved. ${ }^{4}$
Matsuura et $a \bar{l}$ reported that a serum protein, $\beta 2$-glycoprotein I ( $\beta 2$-GP I) with high affinity for anionic phospholipid, is required for binding of aCLs to cardiolipin in autoimmune disease, whereas this requirement is not found in the binding that occurs in association with various infections. In addition, Hunt et al confirmed that binding of aCLs from patients with infections, unlike that of patients with SLE, is not dependent on $\beta 2-G P$ I. Classification of aCLs into $\beta 2-G P$ I dependent and $\beta 2-G P$ I independent has been applied to patients with different clinical manifestations and helped to distinguish between aCLs associated with autoimmune disease and those resulting from infections. ${ }^{7}$ An enzyme linked immunosorbent assay (ELISA) using cardiolipin as antigen has been widely used for detection of aCLs, and the use of adult bovine serum as blocking agent and sample diluent was considered to be the source of $\beta 2-G P$ I. Moreover, it has been reported that aCLs detected with this assay include both $\beta 2-$ GP I dependent and $\beta 2-G P$ I independent forms. ${ }^{5}$ Arvieux et al reported that certain aCLs can bind directly to $\beta 2-G P$ I immobilised on irradiated microtitre plates in the absence of cardiolipin. In addition, an excellent correlation between aCL titres and $\beta 2-G P$ I antibody has been reported, ${ }^{9}{ }^{10}$ giving rise to the idea that autoimmune aCLs and $\beta 2-G P$ I antibodies are one and the same. It was recently reported that these antibodies provide only additional information and that the $\beta 2-G P$ I antibody test should not be considered a substitute for conventional LA or aCL assays. ${ }^{11}$ Moreover, it was reported that $\beta 2-G P$ I antibodies have been closely associated with venous thrombosis but not with other clinical features of APS, such as arterial thrombosis and recurrent abortion. Unlike aCLs, which are often found in several clinical conditions other than APS, ${ }^{1} \beta 2-G P$ I antibodies are seldom found in patients with SLE and without APS and are rare in infectious conditions. ${ }^{12}$

A variety of infectious diseases, particularly viral, such as those caused by HIV-1, parvovirus B19, hepatitis A virus (HAV) and hepatitis $\mathrm{C}$ virus (HCV), have been reported to be associated with the presence of aCLs. ${ }^{13-16}$ However, in some of these reports, aCL isotype distribution and cofactor dependence was not investigated.

In this study, we report the occurrence of $\beta 2-G P$ I dependent and $\beta 2-G P$ I independent aCL isotypes in a number of patients with infection caused by HIV-1, HAV, HCV, or hepatitis $\mathrm{B}$ virus (HBV), as well as an analysis of the relevance of one or more isotypes of aCL in these infectious diseases. 


\section{Patients and methods}

PATIENTS

Stored non-heat-inactivated sera were obtained from the following groups of patients, none of whom were receiving antiviral treatment: group 1, 40 patients with acute HAV infections, confirmed by specific IgM antibodies detected using an ELISA from Abbott (Chicago, Illinois, USA); group 2, 40 patients who tested positive for antibody against $\mathrm{HCV}$ in the second generation ELISA 3.0 (Abbott), confirmed by the RIBA-3.0 assay (Ortho Diagnostic, Raritan, New York, USA); group 3, 40 patients with acute and chronic HBV who tested positive to hepatitis $\mathrm{B}$ surface antigen (HBsAg) in an ELISA (Abbott); 22 of these patients were positive and 18 were negative for anti-HBc IgM detected by ELISA (Abbott); group 4, $40 \mathrm{HIV}-1$ infected patients confirmed by western blot assay (Organon Teknika, Durham, North Carolina, USA). All the above patients were negative for fluorescent Treponema Pallidum antibody (FTA-Abs, Biocientifica, Argentina).

Serum samples from patients with long standing syphilis who were positive for the screening Venereal Disease Research Laboratory (VDRL) test were included in this study and confirmed by FTA-Abs, but none experienced clinical events suggesting APS and HAV, HIV-1, HBV or HCV infections. Other patients with primary and secondary APS (previously reported by our group ${ }^{19}$ ) were also included. The former group of patients provided a positive control for aCL cofactor independence and the second group a positive control for aCL cofactor dependence.

The normal control group consisted of 60 healthy subjects (36 women and 24 men) matched for age and sex. All showed normal coagulation assays and negative serological tests for syphilis VDRL and for the viral infections included in this study.

ASSAY OF ACL COFACTOR DEPENDENCE

The improvement of the aCL ELISA by the use of bovine serum based solutions has been documented previously, and is now considered to be due mainly to the presence of bovine $\beta 2-G P ~ I .{ }^{17}$ To determine cofactor or $\beta 2-G P$ I dependence of aCL binding to cardiolipin, we used a standard ELISA ${ }^{18}$ with minor modifications. ${ }^{19}$ In brief, wells of ELISA plates (LinbroTitertek; ICN-Biomedical, Horsham, Philadelphia, USA) were coated by the addition of 50 $\mu \mathrm{l} /$ well of $30 \mu \mathrm{g} / \mathrm{ml}$ cardiolipin (Sigma Chemical Co, St Louis, Missouri, USA) in ethanol, and the ethanol evaporated at $4^{\circ} \mathrm{C}$ overnight. Plates were washed three times with phosphate buffered saline (PBS) and blocked for one hour by the addition of $100 \mu \mathrm{l} /$ well $10 \%$ (v/v) adult bovine serum (ABS) in PBS (10\% ABS/PBS). After being blocked, plates were washed three times with PBS. The unknown samples and IgG, IgM and IgA aCL calibrators (Louisville APL Diagnostic Inc, Louisville, Kentucky, USA) and our own control sera diluted 1:50 in $10 \% \mathrm{ABS} / \mathrm{PBS}$ were added $(50 \mu \mathrm{l})$ to the ELISA plate in duplicate wells. The diluted serum samples and standards were then incubated for three hours. The samples were then discarded and the plates rinsed three times with PBS. Then $50 \mu \mathrm{l} /$ well alkaline phosphatase labelled, affinity purified, goat antihuman IgG, IgM, or IgA (Sigma Chemical Co) diluted $1: 1000$ in 10\% ABS/PBS was added. After 90 minutes of incubation, the plates were washed three times with PBS, and $100 \mu \mathrm{l} /$ well enzyme substrate ( $p$-nitrophenyl phosphate/ diethanolamine buffer) was added, and the plates were incubated in an oven at $37^{\circ} \mathrm{C}$. The enzyme reaction was stopped with $3 \mathrm{M} \mathrm{NaOH}$ ( $50 \mu \mathrm{l} /$ well) when the highest positive aCL calibrator for that isotype had reached an absorbance at $405 \mathrm{~nm}$ of about 1.0 - for example, for aCL IgG the reaction was stopped when G1=90.1 GPL units reached an absorbance reading of 1.0. Plates were then read in an EL301 microwell strip reader (Bio-Tek Instrument Inc, Winooski, Vermont, USA). Results were expressed as antiphospholipid units (GPL, MPL, and APL for IgG, IgM, and IgA isotypes respectively) using a calibration curve constructed by plotting absorbance against concentration of a given aCL isotype in a log-log equation. A sample with known aCL concentrations was assayed on each occasion to confirm the reliability of the assay. Values higher than 10.2 GPL, 11.5 MPL, and 12.5 APL (mean of 60 healthy sera +2 SDs) were considered positive. Intra-assay and interassay coefficients of variation were $8 \%$ and $10 \%$ respectively.

MODIFIED ELISA FOR COFACTOR INDEPENDENT ACL ISOTYPES

The modified ELISA for aCL cofactor independent isotypes was performed in the absence of ABS as $\beta 2-G P$ I source and at a 1:200 dilution of test samples and controls. We used $2 \%$ ovalbumin (Sigma Chemical Co) as blocking agent and sample diluent instead of bovine serum albumin to avoid possible contamination with bovine $\beta 2-G P$ I. Therefore the assay essentially measures $\beta 2-G P$ I independent binding to the phospholipid surface. The dilution was selected to prevent the cofactor effect of endogenous $\beta 2-\mathrm{GP} I$ in serum samples, since no, or almost no, $\beta 2-\mathrm{GP}$ I dependent aCL will be detected under these experimental conditions. ${ }^{20}$ Results were expressed as the mean of two replicates; a result was regarded as positive when the absorbance was higher than 2 SDs of the mean for the control group. Sera from patients with syphilis or primary or secondary APS, and from selected normal controls were tested in each plate as positive and negative controls respectively. To avoid false positive results due to non-specific binding, a sample in the $\beta 2$-GP I dependent assay had to show a $10 \%$ higher absorbance value than the $\beta 2$-GP I independent assay performed in parallel to be considered positive, and vice versa. ${ }^{20}$ The interassay and intra-assay coefficients of variation were lower than $12 \%(n=8)$ and $8 \%(n=8)$ respectively. 
Table 1 Presence of $\operatorname{Ig} G, \operatorname{Ig} M$, and IgA cofactor dependent and independent antibodies to cardiolipin in patients with viral infections

\begin{tabular}{lllll}
\hline & \multicolumn{3}{l}{ Cofactor dependent/cofactor independent } \\
\cline { 2 - 5 } Antibody isotype & $\begin{array}{l}H I V-1 \\
(n=40)\end{array}$ & $\begin{array}{l}H A V \\
(n=40)\end{array}$ & $\begin{array}{l}H B V \\
(n=40)\end{array}$ & $\begin{array}{c}H C V \\
(n=40)\end{array}$ \\
\hline IgG alone & $0 / 0$ & $0 / 0$ & $1 / 1$ & $0 / 0$ \\
IgM alone & $0 / 0$ & $1 / 22$ & $2 / 5$ & $0 / 0$ \\
IgA alone & $4 / 3$ & $0 / 0$ & $0 / 4$ & $1 / 4$ \\
IgG + IgM & $0 / 0$ & $0 / 1$ & $0 / 1$ & $0 / 0$ \\
IgG + IgA & $4 / 6$ & $0 / 0$ & $0 / 1$ & $0 / 1$ \\
IgM + IgA & $0 / 1$ & $0 / 9$ & $0 / 2$ & $0 / 1$ \\
IgG + IgM + IgA & $0 / 1$ & $0 / 4$ & $0 / 0$ & $0 / 0$ \\
Total No with IgG & $4 / 7$ & $0 / 5$ & $1 / 3$ & $0 / 1$ \\
Total No with IgM & $0 / 2$ & $1 / 36$ & $2 / 8$ & $0 / 1$ \\
Total No with IgA & $8 / 11$ & $0 / 13$ & $0 / 7$ & $1 / 6$ \\
\hline
\end{tabular}

Cofactor dependence was considered positive when there was a $\geqslant 10 \%$ increase in the absorbance reading in the presence of adult bovine serum above those for the same serum samples in the presence of ovalbumin. Cofactor independence was considered positive if there was a reduction in absorbance $\leqslant 10 \%$. $\mathrm{HAV}=$ hepatitis $\mathrm{A}$ virus; $\mathrm{HBV}=$ hepatitis $\mathrm{B}$ virus; $\mathrm{HCV}=$ hepatitis $\mathrm{C}$ virus.

STATISTICAL ANALYSIS

All variables studied are expressed as the arithmetical mean (SEM). Differences in mean values were evaluated by unpaired Student's $t$ test with $\mathrm{p}<0.05$ considered to be significant. The relation between aCL levels and disease activity was studied by the $\chi^{2}$ test.

\section{Results}

Serum samples from 160 patients with acute or chronic viral infection were screened for aCL isotypes, and the cofactor requirement was evaluated. Table 1 shows the prevalence of aCLs in the population studied. Positive samples for aCL (IgG, IgM, or IgA) were found in 19 of 40 patients with HIV-1 infection $(47 \%)$, in 37 of 40 patients with acute HAV $(92 \%)$, in 17 of 40 patients with acute and
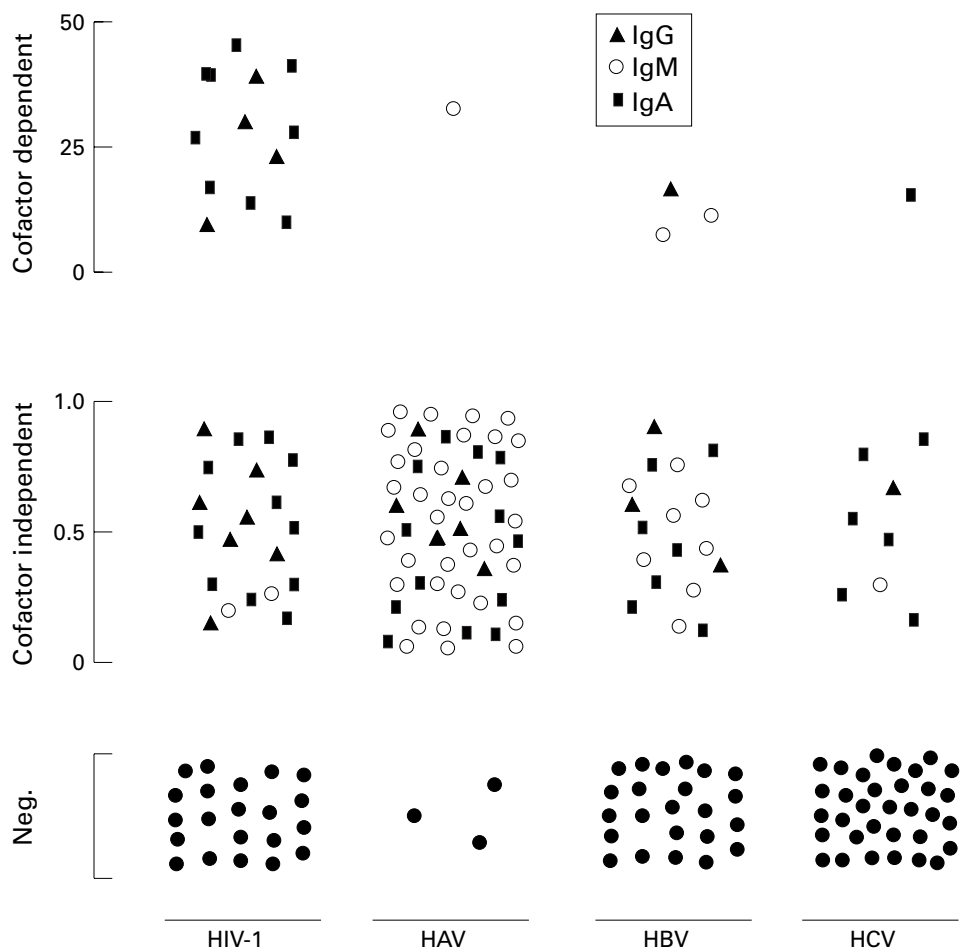

$\mathrm{HCV}$

Figure 1 Distribution and cofactor dependence of the cardiolipin antibody isotypes in the population studied. chronic HBV (42\%), and seven of 40 patients with HCV infection (17\%). The isotype distribution of aCL reactivity also differed among the groups of patients studied (table 1). The total sum of aCLs was naturally higher than the positive samples because several patients exhibited positive titres for more than one isotype.

In the group of patients with HIV-1, the isotype spectra varied: IgA was present in seven of 19 patients, IgG + IgA aCL isotypes in 10 of 19 patients, and two other patients exhibited different combinations of aCL isotypes. Detailed analysis of the results showed that $64 \%$, $100 \%$, and $58 \%$ of the positive samples for IgG, IgM, and IgA aCL isotypes respectively were $\beta 2$-GP I independent as defined by a reduction in binding to cardiolipin (expressed as a decrease in absorbance readings for the same serum samples in the presence of ovalbumin compared with ABS) $\geqslant 10 \%$.

On the other hand, in patients with acute $\mathrm{HAV}$, the $\operatorname{IgM}$ isotype alone was present in 23 of 37 positive patients, combined with IgA in nine of 37 samples, and with IgG + IgA in four of the 37 patients. Interestingly, in this group of patients $100 \%$ of IgG and IgA aCL isotypes and $97 \%$ of the IgM class were $\beta 2-G P$ I or cofactor independent (table 1, fig 1).

In the group with acute and chronic HBV infection, the aCL positive samples for one or more isotype showed a randomised distribution pattern. Three of four positive samples for IgG aCL, eight of 10 positive samples for IgM $\mathrm{aCL}$, and seven of seven IgA aCL isotypes were found to be $\beta 2-$ GP I independent in the modified ELISA. No trend or particular association with the spectra of aCL isotype distribution was found in patients with acute or chronic HBV infection.

Finally, in the patients with HCV, the IgA isotype alone was present in five of the seven positive samples and combined with IgG aCL (one patient) and with $\operatorname{IgM~aCl}$ isotype in the other patient. All samples with $\operatorname{IgG}$ and $\operatorname{IgM}$ isotypes $(100 \%)$ and six of seven samples $(86 \%)$ with IgA aCL were tested as $\beta 2-G P$ I independent.

The complete clinical records were analysed with a specific focus on aCL related involvement such as venous and/or arterial thrombosis and spontaneous abortion. The mean titre of cofactor dependent IgG aCLs was low in all groups but tended to be significantly higher in patients with HIV-1 than in the patient with HBV (28.2 (1.8) v 17.6; p<0.05). For IgM aCLs, the titres were low and there were no differences between patients with HAV and those with HBV (11.6 (0.9) v 12.2 (1.4); NS) and the isotype IgA, $\beta 2$-GP I dependence was higher (38.2 (2.1) APL) in patients with HIV-1 than in the patient with HCV (14.8 APL, $\mathrm{p}<0.05)$.

On the other hand, the aCL values were related to the clinical status at the time the sample was drawn from patients with $\mathrm{HBV}$, but there was no relation between disease activity (acute or chronic) and increased aCL levels. 


\section{Discussion}

Although raised levels of aPLs were first reported in patients with autoimmune diseases, their presence is now known to be more widespread. ${ }^{1}$ Elevated serum levels of all three aCL isotypes, $\operatorname{IgG}, \operatorname{IgM}$, and $\operatorname{IgA}$, have been reported in human infections caused by bacteria, viruses, spirochetes, and parasites; a higher prevalence of the IgG aCL isotype has been found in syphilis, HIV, tuberculosis, Q fever, Lyme disease, and cytomegalovirus infections. ${ }^{15}$ The mechanism of the aCL response to chronic infection such as that caused by HIV-1 and $\mathrm{HCV}$ is unclear. Infection induced aCLs have been shown to differ from autoimmune aCLs. The former tend to be transient, of low titre, and more often of the $\operatorname{IgM}$ isotype; in contrast, autoimmune aCLs often have highly sustained rather than transient titres, and are more often of the IgG isotype. ${ }^{15}{ }^{21}$ Prieto et $a l^{13}$ reported the presence of IgG aCLs in $22 \%$ of $\mathrm{HCV}$ infected patients. This contrasts with a previous report by Gotoh and Matsuda, ${ }^{22}$ who found significantly higher titres of serum aCLs in patients with HIV-1 than in those with HCV. The different results of viral infection associated with aCLs may be related to the immunoassay system used to detect aCLs in the two laboratories. The former group screened for $\beta 2$-GP I dependent aCLs and the latter group analysed $\beta 2-\mathrm{GP}$ I independent aCLs.

In HBV infection, it has been shown that the cell receptors for lipid components of the HBV envelope include both annexin V and $\beta 2$-GP I, which may act as a trigger mechanism to a possible aCL response. ${ }^{23}$ In turn, for patients with $\mathrm{HAV}$, a response to IgM aCLs has been found but no differentiation with regard to requirement of $\beta 2-G P I$ as cofactor has yet been observed. ${ }^{21}$

In this study, we have assayed cofactor dependent and independent aCLs of the IgG, $\operatorname{IgM}$, and $\operatorname{IgA}$ isotypes in a series of 160 sera from patients with a variety of acute and chronic infections verified by the presence of specific antibody levels, in order to establish the importance, if any, of these antibodies in viral infections.

The prevalence of aCLs in patients with viral infections indicated in table 1 is in agreement with previous reports. ${ }^{1321}$ In our study, we found that the prevalence of IgG aCL cofactor dependence was randomly distributed among the groups of patients analysed. The association of aCLs, usually of the IgG isotype, with thrombosis, recurrent fetal losses, and thrombocytopenia has been reported. ${ }^{14}$ It has been reported that IgG aCL levels higher than 40 GPL are closely associated with venous thrombosis. ${ }^{24}$ Our results show that, in patients with HIV-1 or HAV, such levels are unusual and may explain why none of our patients developed any of the complications related to aPLs. Furthermore, in all our patients, except four with HIV-1 and one with HBV infection, these antibodies were $\beta 2$-GP I independent (table 1 , fig 1).
In our study, we found a significantly higher prevalence of IgM aCLs that was virtually confined to patients with HAV infection and to a minor extent to those with acute and chronic HBV infection. Elevated levels of IgM aCLs have been reported in sera of patients with other infectious diseases, ${ }^{25}$ and their pathogenic significance is uncertain. We observed significantly higher levels of binding to cardiolipin of this isotype, and a high percentage of these antibodies were $\beta 2-G P$ I independent (table 1, fig 1). Cross reactivity between raised levels of IgM-type HAV and cardiolipin antibodies, an increase in non-specific binding in the ELISA, or the presence of low affinity antibodies that may have broad epitope specificity are all plausible explanations for this finding.

Raised levels of IgA type aCLs were found in all the groups of patients studied. Elevated levels of IgA aCLs have also been found in patients with mumps. ${ }^{25}$ Unlike the other two isotypes, IgA aCL positivity was higher in patients with $\mathrm{HBV}$ and $\mathrm{HCV}$ infections than in those with HIV-1 and HAV infections. Like the other isotypes, these antibodies were principally $32-$ GP I independent (table 1 ). Antibodies of the IgA isotype are excreted from mucosa associated lymphoid tissue found in various organs such as gastrointestinal, respiratory, and urogenital tracts, and probably participate in protective mechanisms against infectious microorganisms. Our results show that most of the positive IgA aCLs were cofactor independent, suggesting that autoantibodies of the $\operatorname{IgA}$ isotype are part of a non-specific response to viral infections or the consequence of recent mucosal infections caused by an unidentified pathogen.

Previous studies concluded that aCLs in patients with infections such as malaria, tuberculosis, infectious mononucleosis, and syphilis are mainly cofactor independent. ${ }^{13-16}{ }^{21}$ In our study, we extend such conclusions to patients with viral infections caused by HIV-1, HAV, HBV, and HCV. Similar results were published by Biron $e t a l,{ }^{26}$ who found that a high proportion of patients with HCV and positive aCLs was cofactor independent. Unfortunately, we were unable to perform follow up studies in the patients with HIV-1 or HCV infections because they were included in a protocol treatment with antiviral drugs. Futhermore, a significant increase in the rate of aCL positivity during $\alpha$-interferon treatment has recently been reported. ${ }^{27}$ On the other hand, we were unable to find significant differences in levels and distribution of aCL isotypes in patients with acute or chronic HBV infections. These results clearly indicate that the early $\operatorname{IgM}$ results do not represent a "fixed" antibody response to an ultimate "switch" to IgG aCL isotype. Furthermore, these results may be extended to other viral infections. We found that $\operatorname{IgM}$ and $\operatorname{IgA}$ class antibodies are the most prevalent isotypes in the population studied. Several different mechanisms, which are still only attractive hypotheses, may be advanced. There may be cross reactivity between viral antigens and phospholipids during the immune response to 
infection, leading to the development of antibodies, or it may simply be an epiphenomenon of a pathological process that occurs as a secondary consequence of an infection.

In short, these results suggest that, in patients with viral infection and positive aCLs, assessment of the cofactor dependence appears to be a useful tool for estimating the risk of the clinical manifestations observed in the presence of these antibodies. This assay may be regarded as a useful complement to the highly specific $\beta 2-$-GP I antibody assay in the study of the very heterogeneous syndrome called APS.

$1 \mathrm{McNeil} \mathrm{HP}$, Chestermann CN, Krilis SA. Immunology and clinical importance of antiphospholipid antibodies. Adv Immunol 1991;49:193-280.

2 Galli M, Finazzi G, Barbui T. Antiphospholipid antibodies: predictive value of laboratory test. Thromb Haemost 1997; 78:75-8

3 Mijones BL, McCurdy D. The antiphospholipid syndrome: immunologic and clinical aspects. Clinical spectrum and treatment. J Rheumatol 2000;suppl 58:20-8.

4 Martini A, Ravelli A. The clinical significance of antiphospholipid antibodies. Ann Med 1997;29:159-63.

5 Matsuura E, Igarashi Y, Fujimoto M. Heterogeneity of anticardiolipin antibodies defined by the anticardiolipin cofaccardiolipin antibodies defined by the

6 Hunt JE, McNeil HP, Morgan CG, Crameri RM, Krilis SA A phospholipid $\beta 2$-Glycoprotein complex is an antigen for anticardiolipin antibodies occurring in autoimmune disease but not with infection. Lupus 1992;1:75-8

7 Matsuda J, Sahtoh N, Gohchi K, Tsukamoto M. Distinguishing $\beta 2$-Glycoprotein I dependent (systemic lupus erythematosus type) and independent (syphilis type) anticardiolipin antibody with Tween-20. Br J Haematol 1993;85: 799-802.

8 Arvieux J, Roussel B, Jacob MC, Colomb MG. Measurement of antiphospholipid antibodies by ELISA using B2-Glycoprotein as an antigen. J Immunol Methods 1991; 143:223-9.

9 Sebastiani GD, Galeazzi M, Tincani A, Piette JC Font J, Allegri F, et al. Anticardiolipin and anti-beta2-GPI antibodies in a large series of European patients with systemic lupus erythematosus. Prevalence and clinical associations. European Concerted Action on the Immnunoassociations. European Concerted Action on the Immnu

10 Martinuzzo ME, Forastiero RR, Carreras LO. Anti beta 2-glycoprotein I antibodies: detection and association with 2-glycoprotein I antibodies: detection and asso

11 Detkov G, Gil-Aguado A, Lavilla P, Cuesta MV, Fontan G, Pascual-Salcedo D. Do antibodies to beta2-glycoprotein 1 contribute to the better characterization of the antiphospholipid syndrome? Lupus 1999;8:430-8.

12 Sanmarco M, Soler C, Chistides C, Raoult D, Gerolami V, Bernard D. Prevalence and clinical significance of IgG isotype anti- $\beta 2$-glycoprotein I antibodies in antiphospholipid syndrome: A comparative study with anticardiolipin antibodies. J Lab Clin Med 1997;129:499-506.
13 Prieto J, Yuste JR, Beloqui O, Civeira MP, Riezu J, Aguirre $\mathrm{B}$, et al. Anticardiolipin antibodies in chronic hepatitis C: implications of hepatitis $\mathrm{C}$ virus as the cause of antiphospholipid syndrome. Hepatology 1996;23:199-204.

14 Sthoeger ZM, Fogel M, Smirov A, Ergas D, Lurie Y, Bass D, et al. Anticardiolipin autoantibodies in serum samples and cryoglobulins of patients with chronic hepatitis $\mathrm{C}$ infection. Ann Rheum Dis 2000;59:483-6.

15 Ordi-Ros J, Villarreal J, Monegal F, Sauleda S, Esteban J, Vilardell $M$. Anticardiolipin antibodies in patients with chronic hepatitis $\mathrm{C}$ virus infection: characterization in relation to antiphophoslipid syndrome. Clin Diagn Lab Immunol 2000;7:241-4

16 Loizou S, Cazabon JK, Walport MJ, Tait D, So AK. Similarities of specificity and cofactor dependency in similarities of specificity and cofactor dependency in serum antiphospholipid antibodies from patients with
human parvovirus B19 infection and from those with human parvovirus B19 infection and from those with systemic $103-8$.

17 Giordano P, Galli M, Del Vecchio GC, Altomore M, Norbis $\mathrm{F}$, Ruggeri L, et al. Lupus anticoagulant, anticardiolipin antibodies and hepatitis $\mathrm{C}$ virus infection in thalassaemia. Br J Haematol 1998;102:903-6.

18 Harris EN, Gharavi AE, Patel SP, Hughes GRV. Evaluation of the anti-cardiolipin antibody test: report of an international workshop held 4 April 1986. Clin Exp Immunol 1987;68:215-22.

19 Guglielmone HA, Fernandez EJ. Distribution of lupus anticoagulant and anticardiolipin antibodies isotypes in a population with antophospholipid syndrome. J Rheumatol 1999;26:86-90.

20 Aoki K, Dudkiewicz A, Matsuura E, Novotny M, Kaberlein $G$, Gleicher N. Clinical signicance of $\beta 2$-glycoprotein I-dependent anticardiolipin antibodies in the reproductive utoimmune failure syndrome: correlation with conventional antiphospholipid antibody detection systems. Am J Obstet Gynecol 1995;172:926-31.

21 Loizou S, Walport MJ, Davies KA. The antiphospholipid syndrome in infectious diseases. In: Asherson RA, Cervera R, Piette JC, Schoenfeld YS. The antiphospholipid syndrome. Boca Raton, FL: CRC Press, 1996.

22 Gotoh M, Matsuda J. Human immunodeficiency virus rather than hepatitis $\mathrm{C}$ virus infection is relevant to the rather than hepatitis $C$ virus infection is relevant to the 1995;50:220-2.

23 Neurath AR, Strick N, The putative cell receptors for hepatitis $B$ virus (HBV) annexin $\mathrm{V}$ and apolipoprotein $\mathrm{H}$ bind to lipid components of HBV. Virology 1994;204:475-7.

24 Finazzi G, Brancaccio V, Moia M, Ciaverella N, Mazzucconi G, Schinco P, et al. Natural history and risk factors in 360 patients with antiphospholipid antibodies: a four-year prospective study from the Italian registry. Am J Med 1996;100:530-6.

25 Vaarala O, Palosuo T, Kleemola M, Aho K. Anticardiolipin response in acute infections. Clin Immunol Immunopathol $1986 ; 41: 8-15$

26 Biron C, Andreani H, Blanc P, Ramps J, Guigue N, Larrey $\mathrm{D}$, et al. Prevalence of antiphospholipid antibodies in patients with chronic liver disease related to alcohol or hepatitis C virus: correlation with liver injury. J Lab Clin Med 1998;131:243-50.

7 Leroy V, Arvieux J, Jacob MC, Maynard-Huert M, Baud M, Zarski JP. Prevalence and significance of anticardiolipin anti- $\beta 2$ glycoprotein I and anti-prothrombin antibodies in chronic hepatitis C. Br J Haematol 1998;101:468-74. 